\title{
Production research on micro-arc oxidation of magnesium alloy electrombile hub
}

\author{
Ruili Niu \\ Zhengzhou University of industry technology Zhengzhou 451100 China \\ 43955528@qq.com
}

Keywords: magnesium alloy, electrombile hub, micro arc oxidation, quality.

\begin{abstract}
The film forming mechanism of the ceramic layer on large area magnesium alloy electrombile hub in the silicate electrolyte and the influence of micro-arc oxidation process parameters on the membrane layer quality were studied by means of SEM. The corrosion resistance of the membrane layer was detected. The production practice shows that the compact and hard ceramic layer can be obtained on the surface of magnesium alloy electrombile hub by adopting reasonable micro-arc oxidation process parameters, which has a key role in the corrosion protection of magnesium alloy surface.
\end{abstract}

\section{Introduction}

Most electrombile at home and abroad are configured to aluminum alloy wheels hub, which are much lower than the magnesium alloy in the index of quality, energy saving, shock absorption, noise reduction and vehicle dynamic characteristics ${ }^{[1]}$. Magnesium alloy is $30 \%$ lighter than aluminum alloy; the damping effect is 30 times of its. Replace the aluminum alloy hub with magnesium alloy hub, driving the development of magnesium alloy material exploitation and deep processing technology, to reduce electrombile weight and power consumption, energy conservation and environmental protection; To reduce vibration and noise; to improve ride comfort and dynamic characteristics of electrombile (such as transportation quality each reduce 10\%, energy consumption will be reduced $8 \% \sim 10 \%$ ), but its corrosion resistance is poor and application is seriously limited ${ }^{[2]}$. Commonly, the oxide film formed by chemical oxidation and anode oxidation on magnesium alloy has certain protective effect, but its corrosion resistance, environmental friendliness, appearance and other aspects cannot be satisfactory, be badly in need of the development of new surface treatment. In recent years, people have tried to develop a variety of new technologies, such as micro-arc oxidation technology, better meet the above requirements. Micro-arc oxidation, also known as micro plasma oxidation, is a new technology developed on the basis of common anodic oxidation in recent years. ${ }^{[3]}$ Through the combination of the thermal chemical, plasma chemistry and electrochemistry, using a high energy density, the ceramic oxide film is in-situ formed on the surface of the magnesium alloy, which greatly improve the corrosion resistance of magnesium alloy surface casting surface, has a broad application prospect.

\section{Micro-arc oxidation mechanism}

Micro-arc oxidation technology is a new surface treatment technology of environmental protection, which can in-situ form ceramic layer on the surface of some light metal. Its technological characteristics are obvious, the performance of the surface treatment is outstanding, and it has been favored by people since the technology was invented. The mechanism of surface modification technology is to use the electrochemical method to produce the spark discharge spots on the surface of aluminum, magnesium, titanium and other light metal or its alloy as anode in electrolyte aqueous solution, to obtain metal oxide ceramic layer by the combined action of chemical, plasma chemistry and electrochemistry. 


\section{Research methods and technology}

The research object of this paper is magnesium alloy electrombile hub, higher requirements on the toughness of the alloy, so the selection of AM60B. The initial melting temperature is $468{ }^{\circ} \mathrm{C}$, the melting end temperature is $596{ }^{\circ} \mathrm{C}$, the solid-liquid phase line temperature range is $165^{\circ} \mathrm{C}$. Its chemical composition is shown in Table 1.

Table 1 the chemical composition of AM60B alloy (WB / \%)

\begin{tabular}{ccccccccc}
\hline $\mathrm{Al}$ & $\mathrm{Zn}$ & $\mathrm{Mn}$ & $\mathrm{Si}$ & $\mathrm{Cu}$ & $\mathrm{Ni}$ & $\mathrm{Fe}$ & impurities & residues \\
\hline $5.6 \sim 6.4$ & $\leq 0.20$ & $0.26 \sim 0.5$ & $\leq 0.05$ & $\leq 0.008$ & $\leq 0.001$ & $\leq 0.004$ & 0.02 & $\mathrm{Mg}$ \\
\hline
\end{tabular}

Because of the large surface area of magnesium alloy electrombile hub, general above $0.4 \mathrm{~m}^{2}$, the power supply of micro arc oxidation is bigger. In this paper, the micro arc oxidation process of magnesium alloy hub cast was carried out by using the MAO-300 type digital micro-arc oxidation production device developed by Lanzhou University of Technology. Magnesium alloy hub cast in the electrolyte of sodium silicate and sodium hydroxide by micro arc oxidation method. The specific technological process: Firstly, set the process parameters of oxidation and preparation of alkaline sodium silicate solution. Micro-arc oxidation of magnesium alloy hub cast which has been cleaned and lifted into the electrolytic bath, the time is 15 20 min. then clean 2 4 min in water bath, and seal in the hot water (80, 10 15 min). Finally, Cooling in cold water $2 \mathrm{~min}$, hang out the hub, draining, drying, cleaning and inspecting. Must be sealed after the micro arc oxidation treatment, so that the small holes and a large number of micro cracks formed by micro arc oxidation can be closed, which can prevent oxygen entering and causing oxidation. After the completion of the micro arc oxidation, analyze the film samples which were intercepted from magnesium alloy hub casting. In order to facilitate the test analysis, making the sample into a circular plate, the sample cutting position is chosen for the middle part of the hub. The surface of the oxide film was observed and the sample was measured by scanning electron microscope. By changing the composition of the electrolyte and the process parameters of the device, and so on, the structure and properties of the film can be changed, to get the film and surface quality which are suitable for magnesium alloy electrombile hub.

\section{Effect of process parameters of micro arc oxidation on the film quality}

Through the study and analysis of the sample, Micro-arc oxidation technology is actually the oxidation process of magnesium matrix. Fig. 1 is the change of the dimension of the sample before and after oxidation, among them a is the size increase, that is, the thickness of the ceramic film grows out, $b$ is the depth of oxidation towards interior of the matrix, the interface between a and $b$ is initial surface position of sample, $h$ is the total thickness of the oxide film.

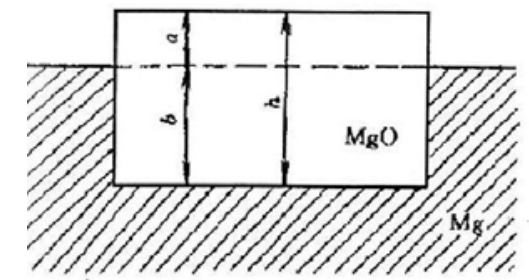

Figure 1 the change of the dimension of the sample before and after the micro arc oxidation

The process parameters such as positive voltage, current density, frequency, duty cycle and oxidation time, etc, have a great influence on experiment. Due to the large surface of magnesium alloy electrombile hub, the micro arc discharge only happen when a certain thickness of oxide film is formed on the surface of the workpiece, therefore, the positive voltage is needn't high, the current is larger, and in the process of formation and thickening of the oxide film, often accompanied by the mutation of current and the positive voltage. When the film thickness reaches a certain degree, it is need to increase the voltage on both ends of the workpiece, usually at around $150 \mathrm{~V}$, the micro arc 
discharge will take place between the workpiece and the electrolyte. As the voltage increases, the current increases, micro-arc density is increasing, arc is more and more bright and micro-arc is constantly beating. At this point the current and voltage are basically linear increase, voltage is about $180 \mathrm{~V}$, and micro arc density basically reached the requirements of the process. At this time, the current growth rate is relatively slow. When the oxide film reaches a certain thickness, even if the voltage increase, the increase of current is small increase, basically tends to be constant, At this time the micro arc on the workpiece is also gradually thin, and the oxidation process can be stopped. If you continue to raise the voltage, the micro arc will be significantly larger, arc light will appear between the workpiece and electrode, lead the pitting corrosion of oxide film, even small cracks appear on the surface of the oxide film, make the workpiece surface quality becomes poor. Micro arc is a kind of electric discharge phenomenon occurring between the oxide film and the inner metal, which is excited by a certain electric field.

The magnitude of current density in a certain extent reflects the degree of micro arc oxidation, which is strongly influence the properties of ceramic layer of micro arc oxidation. The duration of oxidation also seriously affects the corrosion resistance of the film, if too short, although the formation of a dense layer at this time, the film is too thin, not have good corrosion resistance, if too long, after a certain period of time, with the increase of time, although the overall thickness of the film is increasing, the increase is a loose layer, the trend of dense layer is thinning, which is not conducive to the film's corrosion resistance, nor economic. The density of micro arc also related with the pulse frequency, when the pulse frequency increases, the density of micro arc also gradually increased. At this moment, the sudden establishment of a certain electric field will produce a micro arc. In the case of basic technological parameters such as electrolyte concentration, duty cycle, pulse number determined, striking voltage is constant commonly, so when the frequency is increased, the voltage frequency of micro arc increases, and the density of micro arc increases; when the frequency is reduced, the time of zero voltage appears in the unit time will be increased, making the time of the micro arc extinguishing longer, so it is reflected by the decrease of the density of micro arc. After a lot of experiments, the optimum parameters are current density $1.1 \mathrm{~A} / \mathrm{dm} 2$, oxidation time $20 \mathrm{~min}$, frequency $400 \mathrm{~Hz}$, duty cycle $20 \%$.

\section{Structural characteristics of micro arc oxidation coatings}

To intercept specimen from the magnesium alloy hub of micro arc oxidation and observed by Mef3 large metallurgical 1 microscope, the surface morphology of micro-arc oxide film as shown in figure 2. From the graph, we can see that the surface layer of the hub is composed of numerous tiny "small volcano cones" (the projection region of the small hole in the picture), which is a network structure formed by the combination of a branch. "Small volcanic cone" center has a small hole, which is the passage of the micro arc discharge reaction of electrolyte and the matrix, that the spewing channel of the molten oxide when the arc is generated. In addition, the size of the hole is different due to the difference of the current of plasma channel in the micro area, there are a large number of micro cracks around the big hole, and the production of micro cracks is often associated with the stress in the film. Analysis of surface density by analysis software SSM ${ }^{[4]}$, get the sample film $25 \mu \mathrm{m}$, the surface area ratio of the hole is $18 \%$, which illustrates the formation of micro arc oxidation film is good.

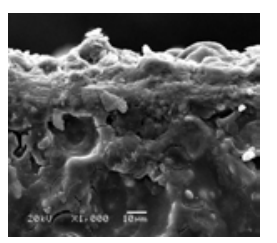

Figure 2 Surface morphology of micro arc oxidation coating on magnesium alloy hub

Figure 3 is the cross section morphology of micro arc oxidation coating through JMS-6700-F field emission scanning electron microscopy (SEM). Figure 3 shows the average film thickness is about 22 
$\mu \mathrm{m}$, oxide film is combined well to the substrate, the decomposition surface is clear and obvious, the surface interface dense, no cavities. From Fig. 3 it can be seen that the micro arc oxidation film is composed of outermost layer of loose layer, the inside of the transition layer and dense layer between the two three parts. Transition layer is the interface between the film and the substrate, holes and other defects existing in the loose layer, dense layer is the key to improve its corrosion resistance ${ }^{[5]}$.

Figure 4is the XRD spectrum of micro-arc oxidation film of AM60B magnesium alloy hub by diffractometer of Phlip X'pert X ray. According to the intensity analysis of the diffraction peak, shows that the peak value of matrix $\mathrm{Mg}$ is obvious, the main phase of micro-arc oxidation film is cubic structure of $\mathrm{MgO}$, The surface containing the spinel phase of $\mathrm{MgAl}_{2} \mathrm{O}_{4}$ and $\mathrm{Mg}_{2} \mathrm{Si}_{2} \mathrm{O}_{4}$, according to the experimental conditions, it may also contain $\mathrm{SiO}_{2}, \mathrm{MgF}_{2}$ and a small amount of $\mathrm{Mg}(\mathrm{OH})_{2}$, and oxides of $\mathrm{Al}, \mathrm{K}, \mathrm{Na}$. Studies show that $\mathrm{MgAl}_{2} \mathrm{O}_{4}$ and $\mathrm{Mg}_{2} \mathrm{Si}_{2} \mathrm{O}_{4}$ can improve the wear resistance of the ceramic layer, $\mathrm{MgO}$ plays a very important role in the corrosion resistance of the ceramic coating. This is the fundamental reason for the performance of the micro arc oxidation film is higher than that of the anodic oxidation film.

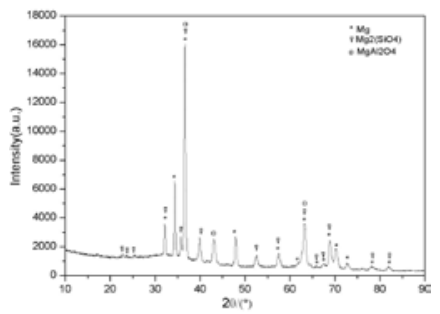

Figure4 the XRD spectrum of micro-arc oxidation film of AM60B magnesium alloy hub

\section{Conclusion}

The process parameters such as positive voltage, current density, frequency, duty cycle and oxidation time, etc, have a great influence on experiment. The optimum parameters are current density $1.1 \mathrm{~A} / \mathrm{dm}^{2}$, oxidation time $20 \mathrm{~min}$, frequency $400 \mathrm{~Hz}$, duty cycle $20 \%$.

The oxide film is divided into two layers, the surface layer and the dense layer. The dense layer is the main body, formed by cubic structure of $\mathrm{MgO}$. The surface layer is $\mathrm{MgO}$ and $\mathrm{MgA} 1_{2} \mathrm{O}_{4}$, spinel phase mixture. The ceramic layer is closely related to the substrate and has a key effect on the surface corrosion of magnesium alloy.

Micro arc oxidation technology is a new type of environmentally friendly surface treatment technology, but for large area of magnesium alloy casting, the time of film formation is longer, production efficiency is low. In order to meet the mass production of large area of magnesium alloy casting, the initial oxidation film can be established by DC power supply in the power supply of micro arc oxidation, the way of strengthening the oxide film by pulsed power supply is respectively carried out. Thus, not only can be obtained the dense and hard ceramic oxide film, but also production efficiency was increased greatly.

\section{References}

[1]Agnew S R, Yoo M H, Tome C N. Application of texture simulation to understanding mechanical behavior of Mg and solid solution alloys containing Li or Y [J].Acta Materialia, 2001, 49: 4277-4289. [2]Agnew S R, Tome C N, Brown D W, et al. Study of slip mechanisms in a magnesium alloy by neutron diffraction and modeling [J].Soripta Materialia, 2003, 48: 1003-1007.

[3]Wang lishi,Cai qizhou. Research conditions on micro arc oxidation of magnesium alloys in foreign countries [J]. Material protection,2004:61-62.

[4]Jiang bailing,Zhang shufen. The study of the corrosion resistance of the ceramic coating formed by micro-arc oxidation on the Mg-base alloy [J]. journal of Chinese society for corrosion and protection,2002:300-303.

[5]Liu yaohui,Lisong.Micro-arc oxidation of AZ91 die cast magnesium alloys in phosphate electrolyte [J]. foundry,2006:1122-1125. 